\title{
Logahedra: a New Weakly Relational Domain
}

\author{
Jacob M. Howe ${ }^{1}$ and Andy King ${ }^{2,3}$ \\ 1 Department of Computing, City University London, EC1V 0HB, UK \\ 2 Portcullis Computer Security Limited, Pinner, HA5 2EX, UK \\ ${ }^{3}$ Computing Laboratory, University of Kent, Canterbury, CT2 7NF, UK
}

\begin{abstract}
Weakly relational numeric domains express restricted classes of linear inequalities that strike a balance between what can be described and what can be efficiently computed. Popular weakly relational domains such as bounded differences and octagons have found application in model checking and abstract interpretation. This paper introduces logahedra, which are more expressiveness than octagons, but less expressive than arbitrary systems of two variable per inequality constraints. Logahedra allow coefficients of inequalities to be powers of two whilst retaining many of the desirable algorithmic properties of octagons.
\end{abstract}

\section{Introduction}

Polyhedra are used in abstract interpretation [4] and model checking real-time [9] and hybrid systems [7]. The domain operations of general polyhedra can be prohibitively expensive, thus there has been much recent interest in the so-called weakly relational domains that seek to balance expressivity and cost by imposing restrictions on the class of inequalities that can be represented. For example, octagons [11] restrict polyhedra [4] to inequalities of at most two variables where the coefficients are -1, 0 or 1 and thereby obtain (at worst) cubic domain operations. Other weakly relational domains whose operations reside in low complexity classes are pentagons [10], two variable per inequality (TVPI) constraints [17] and bounded differences [9]. Domains that do not impose the two variable per inequality restriction include octahedra [3] and template constraints [14].

This paper introduces a new class of weakly relational domain called logahedra. A logahedron is a system of implicitly conjoined two variable inequalities where the coefficients are constrained to be powers of two (or zero). Such coefficients naturally arise because the size of primitive types. For instance, suppose an array of 32-bit integers was dynamically allocated with, say, malloc $(n)$ where $n$ is the size of the memory block in bytes. Then an array index $i$ is in range iff the logahedral inequalities $0 \leq i$ and $4 i+4 \leq n$ are satisfied. Logahedra are proposed as a solution to two problems arising in program analysis. The first problem is that octagons, whilst having good computational properties, are restricted in what they can describe. The second problem is that when the coefficients of inequalities are not constrained (as they are for octagons), for example in general polyhedra or TVPI, the coefficients can easily become very large, requiring 
multiple precision libraries for their storage and manipulation. This can be prohibitively costly [16]. Logahedra address the first problem by allowing a greater variety of constraints to be expressible than octagons, whilst retaining octagons' good computational properties (indeed, logahedra are a true generalisation of octagons; logahedra are strictly more expressive, with octagons being a special case). They address the second problem by restricting the possible coefficients of inequalities; further, since the allowable coefficients are powers of two, they can be represented by their exponents rather than by the number itself, allowing very large coefficients to be represented using machine integers.

Logahedra are themselves a strict subset of TVPI constraints and inherit many of their domain operations. Yet the most important domain operation, (full) completion, has the same complexity as for octagons hence is more efficient than for TVPI, being (truly) cubic. The most complicated domain operation, as with TVPI, is incremental completion. This is the operation of adding a single constraint to an already complete system to give an updated complete system. Incrementally adding constraints is more in tune with the needs of analysis than full closure, therefore incremental closure is arguably the key operation. This operation is also the most complicated and is synthesised from the way new inequalities can be derived in the act of completing a system. This result is applicable to arbitrary two variable systems, not just logahedra. The paper advances the theory of weakly relation domains by making the following contributions:

- The class of logahedral constraints is introduced and it is argued that they have representational advantages over TVPI constraints, whilst being more expressive than octagons.

- A parameterised subclass of logahedra, bounded logahedra, is defined that is a generalisation of octagons in that octagons are a special case of bounded logahedra. Bounded logahedra are more expressive than octagons, whilst retaining their asymptotic complexity.

- Domain operations for both logahedral and bounded logahedral constraints are defined and algorithms for the operations are presented. In part, these build on TVPI operations and include original approaches to completion and abstraction that are applicable to other weakly relational domains.

- Preliminary experiments (and an example) indicate that logahedra have the potential to significantly increase the power of analysis.

\section{Logahedral Constraints}

Logahedra fall between octagons [11] and TVPI [17] in that octagonal constraints can be expressed as logahedral constraints which, in turn, can be expressed as TVPI constraints, that are themselves two variable restrictions of polyhedral constraints. These classes are defined over a set of (indexed) variables $X$ :

Definition 1. Oct $=\{a x+b y \leq d \mid x, y \in X \wedge a, b \in\{-1,0,1\} \wedge d \in \mathbb{Q}\}$

Definition 2. $\log =\left\{a x+b y \leq d \mid x, y \in X \wedge a, b \in\left\{-2^{n}, 0,2^{n} \mid n \in \mathbb{Z}\right\} \wedge d \in \mathbb{Q}\right\}$ 
Definition 3. TVPI $=\{a x+b y \leq d \mid x, y \in X \wedge a, b, d \in \mathbb{Q}\}$

Definition 4. Poly $=\left\{\sum_{i=1}^{|X|} a_{i} x_{i} \leq d \mid x_{i} \in X \wedge a_{i}, d \in \mathbb{Q}\right\}$

Both Oct and TVPI, like Poly, are closed under variable elimination, that is, if $y \in X$ and $S \subseteq$ Oct (respectively $S \subseteq$ TVPI) then $\exists y . S \in$ Oct (respectively $\exists y . S \in \mathrm{TVPI}$ ). For instance, if $S=\{x-2 y \leq 5,3 y+z \leq 7,5 y-u \leq 0\} \subseteq$ TVPI then $\exists y . S$ can be derived by combing pairs of inequality with opposing signs for $y$ to obtain $\exists y . S=\{3 x+2 z \leq 29,-2 u+5 x \leq 25\}$ which is indeed in TVPI. Variable elimination (projection) is an important operation in program analysis and an abstract domain should ideally be closed under it so as to minimise loss of information; Log possesses this property. Furthermore, as well as being more expressive than Oct, Log has representational advantages over TVPI. This makes Log a natural object for study.

\subsection{Representation of Coefficients}

The representational advantages of logahedra are hinted at by their name. Since the absolute value of the coefficients are powers of two, it suffices to represent the logarithm of the value, rather than the value itself. This allows coefficients to be presented by their exponents in machine words, thereby avoiding the computational burden of large coefficients.

As with TVPI inequalities, a logahedral inequality can be binary, that is, involve two variables, when $a \neq 0$ and $b \neq 0$; or be unary, when either $a=0$ or $b=0$ (but not both); or be constant when $a=b=0$. Constant constraints are written as either true or false. A dense representation for the binary case can be achieved by observing that $a x+b y \leq d$ can be expressed as $x+(b / a) y \leq d / a$ if $a>0$ and $-x-(b / a) y \leq-d / a$ otherwise. Thus to represent $a x+b y \leq d$ it is sufficient to distinguish the variables $x$ and $y$, represent the signs of $a$ and $b$ (as two bits) and then either represent $\lg |b / a|$ and $d / a$ or $\lg |b / a|$ and $-d / a$. A unary inequality such as $a x \leq d$ can be expressed as $x \leq d / a$ if $a>0$ and $-x \leq-d / a$ otherwise, therefore it is not even necessary to represent a logarithm. Henceforth, without loss of generality, all logahedra will be represented with first coefficient of $-1,0$ or 1 .

\subsection{Representation of Constants}

Unlike TVPI, that can alternatively be defined with $a, b, d \in \mathbb{Z}$, the constant $d$ is required to be rational, even without a restriction on the first coefficient. Consider, for example, $4 x-2 y \leq 2 / 3$. This has no equivalent logahedral representation with an integer constant. Furthermore, rational constants are required for closure under variable elimination. Consider eliminating $y$ from a system such as $\{x-2 y \leq 3, x+4 y \leq-1\}$ which yields the single inequality $3 x \leq 5$. This, in itself, is not logahedral but the constraint can be equivalently expressed as $x \leq 5 / 3$ which is logahedral. 


\subsection{Bounded Logahedra and their Representation}

The Log class contains an unbounded number of inequalities for each two variable pair. The Oct class, however, is bounded since the size of the coefficients is bounded. Therefore it is worth considering restricting the coefficients of logahedra to within a bound:

Definition 5. $\log _{k}=\left\{a x+b y \leq d \mid x, y \in X \wedge a \in\{-1,0,1\} \wedge b \in C_{k} \wedge d \in \mathbb{Q}\right\}$ where $C_{k}=\left\{-2^{n}, 0,2^{n} \mid n, k \in \mathbb{Z} \wedge-k \leq n \leq k\right\}$.

Notice that Oct $=\log _{0}$. An alternative definition would restrict the first coefficient to be a power of two rather than a unit. However, it is curious to observe that the class $\log _{3}$ is not expressible with the alternative definition.

The case for $\log _{k}$ is further motivated by considering the inequalities required to describe relationships between values stored in machine integers. The following proposition states that inside a bounded box (induced by the size of the type) the set of integer points described by a Log constraint can also be described by a $\log _{k}$ constraint where $k$ is determined by the size of the bounding box.

Proposition 1. Suppose $a x+b y \leq d \in \log$ and $\operatorname{Box}_{k}=\left(\left[-2^{k}, 2^{k}-1\right] \cap \mathbb{Z}\right)^{2}$. Then there exists $a x+b^{\prime} y \leq d^{\prime} \in \log _{k+1}$ such that $\left\{\langle x, y\rangle \in \operatorname{Box}_{k} \mid a x+b y \leq d\right\}$ $=\left\{\langle x, y\rangle \in \operatorname{Box}_{k} \mid a x+b^{\prime} y \leq d^{\prime}\right\}$

Proof. Wlog suppose $c \equiv a x+b y \leq d \in \log$ where $a \in\{-1,1\}$ and $b \notin C_{k+1}$.

Find $\left\langle x^{*}, y^{*}\right\rangle \in \mathbb{Z}^{2}$ that minimises $a x+b y$ subject to $a x+b y \leq d,-2^{k} \leq$ $x \leq 2^{k}-1$ and $-2^{k} \leq y \leq 2^{k}-1$. (This can be achieved in $O(\lg |b|)$ time [5].) The Log constraint $a x+b y \leq d^{\prime}$, where $d^{\prime}=a x^{*}+b y^{*}$, describes the same set of integer points in $\mathrm{Box}_{k}$ as $c$. Put $s=\operatorname{sign}(b), l=l g(|b|), k^{\prime}=\operatorname{sign}(l)(k+1)$ and $b^{\prime}=s 2^{k^{\prime}}$. Then $c^{\prime}:=\left(a x+b^{\prime} y \leq d^{\prime}\right) \in \log _{k+1}$.

If $b^{\prime}=s 2^{k+1}$ then $c^{\prime}$ adds no new integer solutions since Box ${ }_{k}$ has height $2^{k+1}-1$ and $c^{\prime}$ passes through $\left\langle x^{*}, y^{*}\right\rangle$. Likewise, if $b^{\prime}=s 2^{-(k+1)}$ then again $c^{\prime}$ adds no new solutions since Box $x_{k}$ has width $2^{k+1}-1$ and $c^{\prime}$ passes through $\left\langle x^{*}, y^{*}\right\rangle$. The result follows.

The $\log _{k}$ class has $4(2 k+1)$ binary and 4 unary inequalities for each pair of variables, therefore, for fixed $k$, the domain is bounded. The force of the proposition is that for an signed integer representation of, say, 32 bits, it is sufficient to restrict attention to $\log _{33}$. For unsigned integers analogous results hold. Importantly, observe that for any given Box $k$, the domain $\log _{k+1}$ retains closure under variable elimination. This is because, by proposition 1 , any inequality (obtained by combining a pair of inequalities) that falls outside $\log _{k+1}$ can be replaced with another drawn from $\log _{k+1}$ without loss of information. A final observation that is potentially exploitable is that for a given Box $_{k}$ and a pair of coefficients, there is a maximum value for the constant beyond which the inequality does not restrict the box. For example, $x+y \leq d$ is vacuous if $2^{k+1}-2 \leq d$. 


\section{$3 \quad$ Worked example}

This section contains an example to demonstrate the use of logahedra in value range analysis. It serves to illustrate the domain operations required, definitions and algorithms for which will be given in the next section. In addition, the example illustrates the expressivity of logahedra versus octagons.

In the following $\mathrm{C}$ program, read_value() reads a value from a file. The objective of the analysis is to verify the safety of the array access in line 5, no matter what values are read. To this end, the set of $\langle x, y\rangle$ values that can arise immediately after executing lines (1) ...(6) are over-approximated by the logahedra $P_{1}, \ldots, P_{6}$. Each of the these logahedra are defined by a separate equation. The set $P_{2}^{\prime}$ over-approximates the set of $\langle x, y\rangle$ values occurring immediately before line 2. $P_{2}^{\prime}$ differs from $P_{2}$ in that $P_{2}$ assumes that the loop condition holds. The updates at lines (3), (4) and (6) are modelled as translations. Since the value read_value () is not known at analysis time, the values of $\langle x, y\rangle$ at line (5) can be either that at line (3) or (4). $P_{5}$ is thus defined as the join of $P_{3}$ and $P_{4} . P_{2}^{\prime}$ is also formulated as the join of $P_{1}$ and $P_{6}$, but also applies widening, $\nabla$.

(1) int $\mathrm{x}=0, \mathrm{y}=0$, array[8]; $\quad P_{1}=\{\langle 0,0\rangle\}$

(2) while $(x<4)$

$$
\begin{gathered}
\{\quad \text { if }(\text { read_value }()==0) \\
y=y+2 ;
\end{gathered}
$$

$$
\text { else } \begin{array}{r}
y=y+2 ; \\
y=y+1 ;
\end{array}
$$$$
P_{2}^{\prime}=P_{2}^{\prime} \nabla\left(P_{1} \sqcup P_{6}\right) \quad P_{2}=P_{2}^{\prime} \sqcap\{\langle x, y\rangle \mid x<4\}
$$$$
\operatorname{array}[\mathrm{y}]=\mathrm{y} \text {; }
$$

$$
P_{3}=\left\{\langle x, y+2\rangle \mid\langle x, y\rangle \in P_{2}\right\}
$$

$$
\mathrm{x}=\mathrm{x}+1 ;
$$

$$
\begin{aligned}
& P_{4}=\left\{\langle x, y+1\rangle \mid\langle x, y\rangle \in P_{2}\right\} \\
& P_{5}=P_{3} \sqcup P_{4} \\
& P_{6}=\left\{\langle x+1, y\rangle \mid\langle x, y\rangle \in P_{5}\right\}
\end{aligned}
$$

Solutions to the equations, or at least upper-approximations to them, can be found by repeatedly applying the equations until a fixpoint is reached. As in other polyhedral analyses [4], widening is introduced to enforce convergence since $P_{1}, P_{2}^{\prime}, \ldots, P_{6}$ grow as the equations are reapplied. To obtain convergence, it is sufficient to put $Q_{1} \nabla Q_{2}=Q_{1} \sqcup Q_{2}$ if $Q_{1}$ and $Q_{2}$ differ in dimension; otherwise $Q_{1} \nabla Q_{2}$ is defined as the (non-redundant) inequalities of $Q_{1}$ that hold for $Q_{2}$. This removes unstable bounds from $Q_{1}$ whilst ensuring $Q_{1} \sqcup Q_{2} \subseteq Q_{1} \nabla Q_{2}$ [6].

The diagrams in Fig. 1 show how $P_{1}, P_{2}^{\prime}, \ldots, P_{6}$ develop during the fixpoint calculation from their initial values of $\emptyset$. Diagram (a) shows how $P_{1}$ is changed by the first equation; thereafter $P_{1}$ is stable. Initially $P_{6}=\emptyset$ so that $P_{1} \sqcup P_{6}=P_{1}$. Since $P_{2}^{\prime}=\emptyset$ differs in dimension from $P_{1}$, the second equation assigns $P_{2}^{\prime}$ to $P_{1}$. Diagram (c) illustrates $P_{5}$, which is a line segment, that is the join of $P_{3}$ and $P_{4}$ that are themselves translations of $P_{2} . P_{6}$ in diagram (d) is a translation of $P_{5}$. When reapplying the second equation, $P_{2}^{\prime}$ and $P_{1} \sqcup P_{6}$ again differ in dimension so that $P_{2}^{\prime}$ is updated to the solid triangle depicted in diagram (e). Diagrams (f) and (g) illustrate the effect of translations and a join, $P_{5}$, and another translation, $P_{6}$. On the third application of the second equation, $P_{2}^{\prime}$ and $P_{1} \sqcup P_{6}$ are 2 dimensional, hence $P_{2}^{\prime}$ is updated to just retain the two stable inequalities, as illustrated in (i). Diagram (j) shows how the loop condition is 


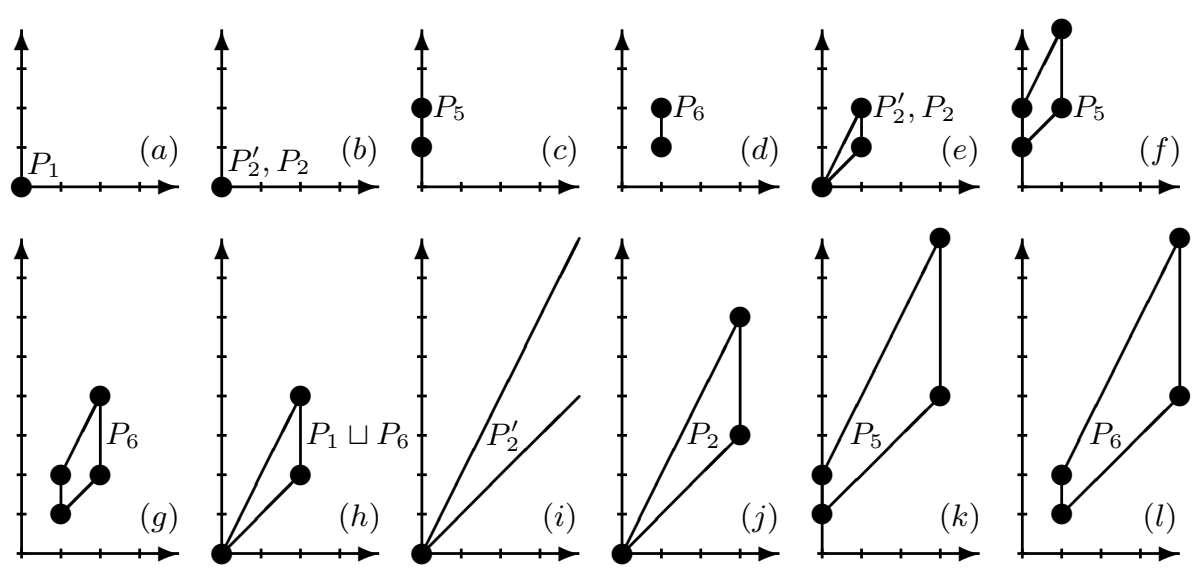

Fig. 1. Logahedra $P_{1}, P_{2}^{\prime}, P_{2}, P_{5}$ and $P_{6}\left(P_{3}\right.$ and $P_{4}$ are omitted $)$

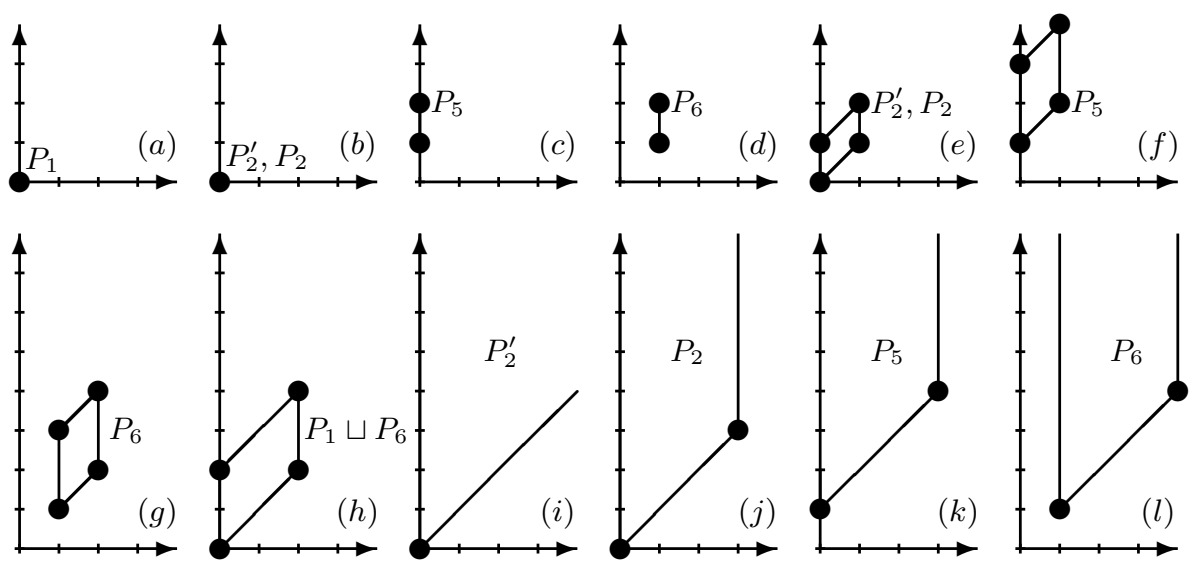

Fig. 2. Octagons $P_{1}, P_{2}^{\prime}, P_{2}, P_{5}$ and $P_{6}\left(P_{3}\right.$ and $P_{4}$ are omitted $)$

reinserted. $P_{2}^{\prime}$ remains unchanged when its equation is applied a fourth time. The $\langle x, y\rangle$ values summarised by $P_{5}$ in diagram $(\mathrm{k})$ show that $y$ can possibly take a value of 8 , indicating a possibly erroneous array access. The analysis shows that enlarging the array by one element alleviates the problem. For this example, the analysis would terminated without widening, though this is not always so.

Fig. 2 repeats the analysis with octagons. Diagrams (e) and (h) show that the upper bound on $y$ is unstable since the domain cannot express $y \leq 2 x$. Hence, in diagram (i), $P_{2}^{\prime}$ loses crucial information on the maximal values of $y$. Moreover, if widening was not applied, then the upper bound of $y$ would grow indefinitely. Thus the loss of information cannot be remedied by more sophisticated widening. Curiously, the example was not manufactured to show the benefits of logahedra, but rather to illustrate polyhedral analysis to a lay audience. 


\section{Logahedral Domain Operations}

This section details the domain operations previously introduced. These operate on finite sets of inequalities, such as $\wp^{f}(\log )$ and $\wp^{f}\left(\log _{k}\right)$, where $\wp^{f}(S)$ denote the set of finite subsets of a given set $S$. The entailment ordering on $\wp^{f}$ (Poly), and its subdomains $\wp^{f}($ TVPI $), \wp^{f}(\log )$ and $\wp^{f}\left(\log _{k}\right)$, is given by $I_{1}=I_{2}$ iff any assignment that satisfies each inequality $c_{1} \in I_{1}$ also satisfies each $c_{2} \in I_{2}$. Equivalence is defined as $I \equiv I^{\prime}$ iff $I \models I^{\prime}$ and $I^{\prime} \mid=I$.

Example 1. If $I=\{x-2 y \leq 7, y \leq 2\}$ and $I^{\prime}=\{x \leq 12\}$ then $I \models I^{\prime}$ since every assignment to $x$ and $y$ satisfying $I$ also satisfies $I^{\prime}$. But $I^{\prime} \not \models I$. If $c:=3 x \leq 2$ and $c^{\prime}:=x \leq 2 / 3$ then $\{c\} \equiv\left\{c^{\prime}\right\}$ and indeed $c$ and $c^{\prime}$ are multiples of one another.

\subsection{Completion}

The algorithms for many operations on logahedra (and other weakly relational domains) require implied inequalities to be made explicit. The process of inferring all implied constraints is called completion and is the dominating computational expense in the operations of which it forms a part. Therefore a clear understanding of how completion is applied, along with efficient algorithms, is essential. Completion is formalised in terms of syntactic projection:

Definition 6. If $Y \subseteq X$ then syntactic projection onto $Y$ is defined $\pi_{Y}(S)=$ $\{c \in S \mid \operatorname{vars}(c) \subseteq Y\}$, where vars $(c)$ is the set of variables occurring in $c$.

Definition 7. The set of logahedral inequalities $I \subseteq \log$ is complete iff for all $c \in \log$ it holds that if $I \models c$ then $\pi_{\operatorname{vars}(c)}(I) \models c$.

Example 2. Let $I_{0}=\{x-y \leq 0,2 x+y \leq 1\}$. $I_{0}$ is not complete because $I_{0} \models x \leq 1 / 3$ but $\pi_{\{x\}}\left(I_{0}\right)=\emptyset \not \models x \leq 1 / 3$. Put $I_{1}=I_{0} \cup\{x \leq 1 / 3\}$. The constant constraint true does not compromise completion since $\pi_{\emptyset}\left(I_{1}\right)=\emptyset \models$ true.

Example 3. Suppose $I_{0}=\{x-y \leq-1, y-z \leq-1, z-x \leq-1\}$. $I_{0}$ is not complete since $\pi_{\{x, z\}}\left(I_{0}\right) \not \forall x-z \leq-2$ and $\pi_{\{x, y\}}\left(I_{0}\right) \not \models y-x \leq-2$. Put $I_{1}=I_{0} \cup\{x-z \leq-2, y-x \leq-2\}$. $I_{1}$ is still not complete since $\pi_{\emptyset}\left(I_{1}\right) \models$ false ( $I_{0}$ is unsatisfiable). Put $I_{2}=I_{1} \cup\{$ false $\}$. Then $I_{2}$ is complete.

The action of deriving implied inequalities, or computing resultants to use the terminology of Nelson [12], is formalised below:

Definition 8. If $c=a x+b y \leq d, c^{\prime}=a^{\prime} x+b^{\prime} z \leq d^{\prime}$ and $a \cdot a^{\prime}<0$ then result $\left(c, c^{\prime}, x\right)=\left|a^{\prime}\right| b y+|a| b^{\prime} z \leq\left|a^{\prime}\right| d+|a| d^{\prime}$ otherwise result $\left(c, c^{\prime}, x\right)=\perp$.

The operation result $\left(c, c^{\prime}, x\right)$ is analogously defined when $c:=a x \leq d$ or $c^{\prime}:=a^{\prime} x \leq d^{\prime}$. Note that it is necessary to stipulate which variable is eliminated because a single pair of inequalities may possess two resultants, as is illustrated by the pair $c:=x+y \leq 1, c^{\prime}:=-2 x-3 y \leq 1$ for which result $\left(c, c^{\prime}, x\right)=-y \leq 3$ and result $\left(c, c^{\prime}, y\right)=x \leq 4$. The resultant operator lifts to sets of inequalities:

Definition 9. If $I_{1}, I_{2} \subseteq$ TVPI then

$$
\operatorname{result}\left(I_{1}, I_{2}\right)=\left\{c \mid c_{i} \in I_{i} \wedge x \in \operatorname{vars}\left(c_{1}\right) \cap \operatorname{vars}\left(c_{2}\right) \wedge c=\operatorname{result}\left(c_{1}, c_{2}, x\right) \neq \perp\right\}
$$


Full completion Completing a set of inequalities $I$ [17] amounts to repeatedly augmenting $I$ with result $(I, I)$ until an $I^{\prime}$ is obtained such that no further (non-redundant) inequalities can be added to $\pi_{Y}\left(I^{\prime}\right)$ for any $Y \subseteq X$. During completion, the computation of resultants is interleaved with the removal of redundant inequalities. An inequality $c$ is considered to be redundant in $I$ iff $\pi_{\operatorname{vars}(c)}(I) \backslash\{c\} \models c$, that is, $c$ is redundant in its syntactic projection $\pi_{\operatorname{vars}(c)}(I)$. To remove such constraints from $I$, the existence of an operator filter $Y(I)=I^{\prime}$ is assumed for each $Y \subseteq X$ such that $|Y| \leq 2$. The operator is assumed to satisfy the three conditions that $I^{\prime} \subseteq I, I^{\prime} \equiv I$ and $I^{\prime \prime} \not \equiv I$ for all $I^{\prime \prime} \subset I^{\prime}$. Such an operator can be constructed straightforwardly, and resides in $O(|I|)[8$, section 2 ]. With filter ${ }_{Y}$ in place, it is possible to filter an entire system $I \subseteq \log$ by computing filter $(I)=\cup\left\{\operatorname{filter}_{Y}\left(\pi_{Y}(I)\right)|Y \subseteq X \wedge| Y \mid=2\right\}$.

Definition 10. The (full) completion operator complete $: \wp(\log ) \rightarrow \wp(\log )$ is defined: complete $(I)=\cup_{i \geq 0} I_{i}$ where $I_{0}=I$ and $I_{i+1}=$ filter $\left(I_{i} \cup \operatorname{result}\left(I_{i}, I_{i}\right)\right)$.

Nelson [12], working over TVPI, used a divide and conquer argument to bound the number of iterations that need be computed before stability is achieved:

Lemma 1. complete $(I)=I_{m}$ where $m=\lceil\lg (|X|)\rceil$ and $I_{m}$ is defined as above.

This result becomes more intruiging when the domain is $\log _{k}$. Completion can be calculated in a semi-naive fashion by defining $I_{0}=I$ and $\delta_{0}=I$ and computing $I_{i+1}=$ filter $\left(I_{i} \cup \operatorname{result}\left(\delta_{i}, I_{i}\right)\right)$ and $\delta_{i+1}=I_{i+1} \backslash I_{i}$ for $i \in[0, m-1]$. Since $\left|\cup_{i=0}^{m} \delta_{i}\right|$ is in $O\left(|X|^{2}\right)$ it follows that the cumulative running time of result $\left(\delta_{i}, I_{i}\right)$ is $O\left(|X|^{3}\right)$. Since each invocation of filter resides in $O\left(|X|^{2}\right)$ and it is called $m-1$ times, it follows that the running time of completion is $O\left(|X|^{3}\right)$. Hence, like Oct, but unlike TVPI, $\log _{k}$ comes with a (full) completion operation that resides in $O\left(|X|^{3}\right)$. It is conceivable that this result extends to other subclasses of TVPI that also retain closure under variable elimination.

Incremental completion During analysis inequalities are encountered one by one. Thus an important addition to full completion is incremental completion that takes a complete system, augments it with an additional inequality and returns the completion of the augmented system. Such an algorithm has been proposed for TVPI [15, Algorithm 7], together with a sketched correctness proof. Given the importance of completion, the following proposition, whose proof is given in [8], provides a rigorous foundation for an incremental algorithm.

Proposition 2. If $c^{\prime} \in \log , I \subseteq \log$ is complete and $c \in \operatorname{complete}\left(I \cup\left\{c^{\prime}\right\}\right)$ then one of the following hold:

$$
\begin{aligned}
& -c \in I \cup\left\{c^{\prime}\right\} \\
& -c \in \operatorname{result}\left(c^{\prime}, c_{0}\right) \text { where } c_{0} \in I \\
& -c \in \operatorname{result}\left(\operatorname{result}\left(c^{\prime}, c_{0}\right),\left\{c_{1}\right\}\right) \text { where } c_{0}, c_{1} \in I
\end{aligned}
$$


$I \cup\left\{c^{\prime}\right\}$ can thus be completed by computing $I_{2}=$ filter $\left(I_{1} \cup \operatorname{result}\left(I_{1} \backslash\left\{c^{\prime}\right\}, I_{1} \backslash I\right)\right)$ where $I_{1}=$ filter $\left(I \cup\left\{c^{\prime}\right\} \cup \operatorname{result}\left(I,\left\{c^{\prime}\right\}\right)\right)$. Nelson showed that if $J_{1}, J_{2} \subseteq$ TVPI where vars $\left(J_{1}\right)=\{x, y\}$ and $\operatorname{vars}\left(J_{2}\right)=\{y, z\}$ then $\left|\operatorname{result}\left(J_{1}, J_{2}\right)\right| \leq 2\left|J_{1}\right|+2\left|J_{2}\right|$ [12, section 3]. It follows that $\left|I_{1}\right| \leq 3|I|+3$ and $\left|I_{2}\right| \leq 13|I|+13$, thus although computing $I_{2}$ for Log takes $O\left(|I|^{2}\right)$ time it requires $O(|I|)$ space overall. For $\log _{k}$ with fixed $k$, computing $I_{1}$ and $I_{2}$ both reside in $O\left(|X|^{2}\right)$. This squares with incremental closure for octagons which is also in $O\left(|X|^{2}\right)$.

\subsection{Entailment}

The value of completeness is that it simplifies other operations. To illustrate, consider the problem of detecting if a fixpoint has been reached, that is, deciding whether $I_{1} \models I_{2}$ for $I_{1}, I_{2} \in \wp^{f}(\log )$. Suppose $I_{1}$ is complete. If false $\in I_{1}$ then it follows $I_{1} \models I_{2}$. Otherwise $I_{1} \models I_{2}$ iff $\pi_{Y}\left(I_{1}\right) \models \pi_{Y}\left(I_{2}\right)$ for all $Y \subseteq X$ and $|Y|=2$. Moreover, recall that inequalities $a x+b y \leq d$ are maintained in the form $a \in\{-1,0,1\}$ and suppose $b \in\{-1,1\}$ if $a=0$. Then the planar entailment check $\pi_{Y}\left(I_{1}\right) \models \pi_{Y}\left(I_{2}\right)$ can be decided by testing filter ${ }_{Y}\left(\pi_{Y}\left(I_{1}\right) \cup \pi_{Y}\left(I_{2}\right)\right)=\pi_{Y}\left(I_{1}\right)$.

\subsection{Variable Elimination}

Variable elimination (projection) is required to remove out of scope variables, and all information pertaining to them, from a logahedral abstraction. FourierMotzkin can be applied to eliminate a variable $x$ from $I \in \wp^{f}(\log )$ which amounts to computing $\exists x . I=\cup\left\{c \mid c=\operatorname{result}\left(c_{1}, c_{2}, x\right) \wedge c_{1}, c_{2} \in I \wedge c \neq \perp\right\}$. However if $I$ is complete then $\exists x . I=\cup\left\{\pi_{Y}(I)|Y \subseteq X \backslash\{x\} \wedge| Y \mid \leq 2\right\}$. If $I$ were not so, then $\exists x . I$ information could be lost as is witnessed by $I=\{w-x \leq 0$, $x-y \leq 0\}$. Then $\cup\left\{\pi_{Y}(I)|Y \subseteq X \backslash\{x\} \wedge| Y \mid \leq 2\right\}=\emptyset$ yet $\exists x . I \models w-y \leq 0$.

Projection also provides a way to realise translations induced by assignments of the form $x=x+c$ where $c \in \mathbb{Z}$. If $I$ describes the state prior to the assignment, then the state after is described by $\exists x^{\prime} .\left(\left\{x=x^{\prime}\right\} \cup \exists x .\left(I \cup\left\{x^{\prime}=x+c\right\}\right)\right)$.

\subsection{Abstraction}

This section explains how to approximate a finite set of arbitrary Poly constraints by a finite set of Log constraints. Abstraction is employed as a component of join but is also used to translate program statements, for example, loop conditions, into logahedral inequalities. Approximation has two stages: projection onto planar sets of TVPI constraints and relaxation of these to logahedral constraints. Consider the latter step first, and suppose $I \subseteq$ TVPI is finite and that vars $(I) \subseteq Y$ where $|Y|=2$. Suppose $I=\left\{c_{0}, \ldots, c_{n-1}\right\}$ is non-redundant and ordered by orientation. This can be achieved in $O(|I| \lg |I|)$ time. Wlog each $c_{i}$ is assumed to take the form $a_{i} x+b_{i} y \leq d_{i}$ where $a_{i} \in\{-1,0,1\}$. Let $c_{1} \angle c_{2}$ be a predicate that holds when $a_{1} b_{2}-a_{2} b_{1}<0$, that is, that $c_{1}$ is oriented before $c_{2}$ in a clockwise relative order by angle. Suppose also that $c_{-1}=c_{n-1}$ and $c_{n}=c_{0}$. If $c_{i}$ and $c_{i+1}$ do not intersect at a vertex let $p_{i}=\perp$, otherwise let $p_{i}=\left(\psi_{i}, \phi_{i}\right)$ be this vertex, 


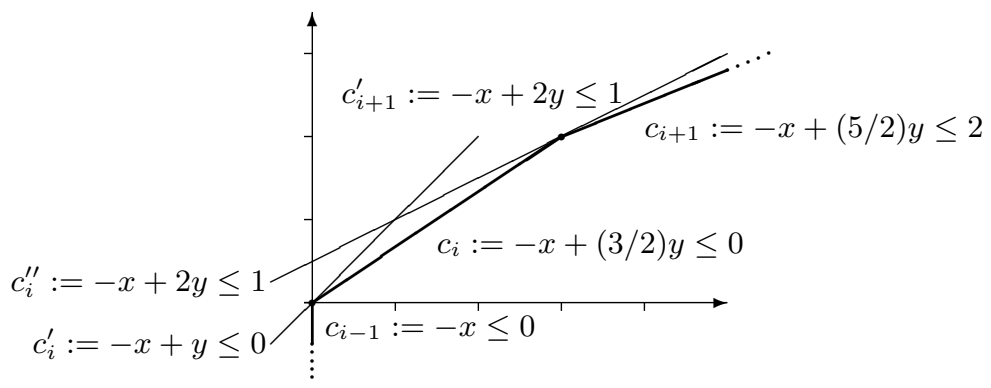

Fig. 3. Approximation with logahedral constraints

which can be calculated in constant time. If $b_{i}=0$ then put $l_{i}=u_{i}=0$, otherwise define $l_{i}=\operatorname{sign}\left(b_{i}\right) 2^{\left\lfloor\lg \left(\left|b_{i}\right|\right)\right\rfloor}$ and $u_{i}=\operatorname{sign}\left(b_{i}\right) 2^{\left\lceil\lg \left(\left|b_{i}\right|\right)\right\rceil}$. For each $i \in[0, n-1]$ put $c_{i}^{\prime}=\perp$ if $p_{i-1}=\perp$ otherwise define

$$
c_{i}^{\prime}= \begin{cases}a_{i} x+u_{i} y \leq a_{i} \psi_{i-1}+u_{i} \phi_{i-1} & \text { if } a_{i}=\operatorname{sign}\left(b_{i}\right) \\ a_{i} x+l_{i} y \leq a_{i} \psi_{i-1}+l_{i} \phi_{i-1} & \text { if } a_{i} \neq \operatorname{sign}\left(b_{i}\right)\end{cases}
$$

Likewise if $p_{i}=\perp$ put $c_{i}^{\prime \prime}=\perp$ otherwise define

$$
c_{i}^{\prime \prime}= \begin{cases}a_{i} x+l_{i} y \leq a_{i} \psi_{i}+l_{i} \phi_{i} & \text { if } a_{i}=\operatorname{sign}\left(b_{i}\right) \\ a_{i} x+u_{i} y \leq a_{i} \psi_{i}+u_{i} \phi_{i} & \text { if } a_{i} \neq \operatorname{sign}\left(b_{i}\right)\end{cases}
$$

The $a_{i}=\operatorname{sign}\left(b_{i}\right)$ test determines whether the inequalities resulting from the upper and lower approximations of $\left|b_{i}\right|$ support $p_{i-1}$ or $p_{i}$. Some of the logahedral constraints $c_{i}^{\prime}, c_{i}^{\prime \prime}$ may be too strong in that $I \not \forall c_{i}^{\prime}$ or $I \not \forall c_{i}^{\prime \prime}$ and these, with the $\perp$ constraints, are filtered out to abstract $I$ as follows:

Definition 11. Let $\alpha_{Y}(I)=\left\{c_{i}^{\prime} \mid c_{i}^{\prime} \neq \perp \wedge c_{i-1} \angle c_{i}^{\prime}\right\} \cup\left\{c_{i}^{\prime \prime} \mid c_{i}^{\prime \prime} \neq \perp \wedge c_{i}^{\prime \prime} \angle c_{i+1}\right\}$ where $c_{i}^{\prime}, c_{i}^{\prime \prime}$ are defined as above for finite $I \subseteq$ TVPI, $\operatorname{vars}(I) \subseteq Y$ and $|Y|=2$.

Note that the filtering test amounts to an $O(1)$ orientation check. The angular test guarantees that the retained inequalities are supporting.

Example 4. The example illustrates the approximation of two constraints from a larger system $I$. Suppose $c_{i-1}:=-x \leq 0, c_{i}:=-x+(3 / 2) y \leq 0, c_{i+1}:=$ $-x+(5 / 2) y \leq 2$ and $p_{i+1}=\perp$. The logahedral approximation is illustrated in Fig. 3. Observe $p_{i-1}=(0,0)$ and $p_{i}=(3,2)$. Since $l_{i}=2^{\lfloor\lg (3 / 2)\rfloor}=1$, $u_{i}=2^{\lceil\lg (3 / 2)\rceil}=2, a_{i} \neq \operatorname{sign}\left(b_{i}\right), p_{i-1} \neq \perp$ and $p_{i} \neq \perp$ it can be seen that $c_{i}^{\prime}:=-x+y \leq 0$ and $c_{i}^{\prime \prime}:=-x+2 y \leq 1$. Moreover, since $l_{i+1}=2^{\lfloor\lg (5 / 2)\rfloor}=2$, $u_{i+1}=2^{\lceil\lg (5 / 2)\rceil}=4, a_{i+1} \neq \operatorname{sign}\left(b_{i+1}\right), p_{i} \neq \perp$ and $p_{i+1}=\perp$ it follows $c_{i+1}^{\prime}:=-x+2 y \leq 1$ and $c_{i+1}^{\prime \prime}:=\perp$.

Now suppose instead, $c_{i+1}:=-x+(7 / 4) y \leq 1 / 2$, which preserves $p_{i}=(3,2)$. Then $I \not \neq c_{i}^{\prime \prime}$ and $c_{i}^{\prime \prime}$ is not an approximating constraint. But $c_{i}^{\prime \prime} \angle c_{i+1}$ does not hold since $(-1.7 / 4)-(-1.2)>0$, hence $c_{i}^{\prime \prime} \notin \alpha_{Y}(I)$. Likewise $c_{i+1}^{\prime} \notin \alpha_{Y}(I)$.

Although $\alpha_{Y}$ is partial, it can be used to abstract arbitrary TVPI systems: 
Definition 12. The abstraction map $\alpha: \wp^{f}($ TVPI $) \rightarrow \wp^{f}(\log )$ is given by $\alpha(I)=\cup\left\{\alpha_{Y}(\bar{\exists} Y . I)|Y \subseteq X \wedge| Y \mid=2\right\}$

In the above $\bar{\exists} Y . I$ denotes project $I$ onto $Y$, that is, the repeated application of Fourier-Motzkin to elimination of all variables $x \in X \backslash Y$ from $I$. However, if $I$ is complete then $\alpha(I)=\cup\left\{\pi_{Y}(I)|Y \subseteq X \wedge| Y \mid=2\right\}$. Interestingly, $\alpha$ can be immediately lifted to $\alpha: \wp^{f}$ (Poly) $\rightarrow \wp^{f}(\log )$ since if $I$ is a finite set then $\bar{\exists} Y . I$ is a finite set. The symbol $\alpha$ hints at the existence of a Galois connection between $\left\langle\wp^{f}\right.$ (Poly), $\left.\models\right\rangle$ and $\left\langle\wp^{f}(\log ), \models\right\rangle$, and indeed $\alpha$ is monotonic. But such a structure can only be obtained by quotienting $\wp^{f}$ (Poly) and $\wp^{f}(\log )$ by $\equiv$ to obtain posets. The upper adjoint of $\alpha$ is the identity.

To abstract the inequalities $I$ or $I \cup \operatorname{Box}_{k}$ to $\log _{k}$ put $c_{i}^{\prime}:=\operatorname{sign}\left(b_{i}\right) y \leq$ $\operatorname{sign}\left(b_{i}\right) \phi_{i-1}$ and $c_{i}^{\prime \prime}:=a_{i} x+\operatorname{sign}\left(b_{i}\right) 2^{k} y \leq a_{i} \psi_{i}+\operatorname{sign}\left(b_{i}\right) 2^{k} \phi_{i}$ if $a_{i}=\operatorname{sign}\left(b_{i}\right)$ and $k<\left\lceil\lg \left(\left|b_{i}\right|\right)\right\rceil$. Likewise, if $\left\lfloor\lg \left(\left|b_{i}\right|\right)\right\rfloor<-k$ then put $c_{i}^{\prime}:=a_{i} x+\operatorname{sign}\left(b_{i}\right) 2^{-k} y \leq$ $a_{i} \psi_{i-1}+\operatorname{sign}\left(b_{i}\right) 2^{-k} \phi_{i-1}$ and $c_{i}^{\prime \prime}:=a_{i} x \leq a_{i} \psi_{i}$. Similarly for $a_{i} \neq \operatorname{sign}\left(b_{i}\right)$.

\subsection{Meet and Abstraction}

Meet over $\left\langle\wp^{f}(\log ), \models\right\rangle$ can be defined by $\left[I_{1}\right]_{\equiv} \sqcap\left[I_{2}\right]_{\equiv}=\left[I_{1} \cup I_{2}\right]_{\equiv}$. Meet thus reduces to set union when a class $[I]_{\equiv}$ is represented by a set $I$. Henceforth quotienting will be omitted, for brevity, and to reflect implementation.

A less trivial problem is that of computing $\alpha\left(I_{1} \cup I_{2}\right)$ where $I_{1} \in \wp^{f}(\log )$ and $I_{2} \in \wp^{f}$ (Poly). This arises as a special case when a statement is encountered, such as a loop condition $x \leq y+z$ or an assignment $x=y+z$, that cannot be expressed logahedrally. If these statements are described respectively by $I_{2}=\{x \leq y+z\}$ and $I_{2}=\{y+z \leq x, x \leq y+z\}$, and $I_{1}$ is a logahedral description of the program state, then the subsequent state is described by $\alpha\left(I_{1} \cup I_{2}\right)$. Thus, in an analysis, $\alpha\left(I_{1} \cup I_{2}\right)$ performs the role of meet when $I_{2}$ is not logahedral.

This problem has been tackled with linear programming [15]. To illustrate, suppose $I_{2}=\left\{c^{\prime}\right\}$ where $c^{\prime}:=\sum_{k=1}^{n} a_{k}^{\prime} x_{k} \leq d^{\prime}$. Let $i, j \in[1, n]$ where $i \neq j$ and minimise $\sum_{k \neq i, k \neq j} a_{k}^{\prime} x_{k}$ subject to $I_{1}$ and $c^{\prime}$. If a minima $d_{i, j}$ exists then it follows $I_{1} \cup\left\{c^{\prime}\right\} \models a_{i}^{\prime} x_{i}+a_{j}^{\prime} x_{j} \leq d^{\prime}-d_{i, j}$. TVPI inequalities found this way are added to $I_{1}$. An alternative and potentially more precise approach, which is applicable to both TVPI and logahedra, is based on extending the resultant operator to polyhedral inequalities in the following fashion:

Definition 13. If $c:=a_{i} x_{i}+a_{j} x_{j} \leq d \in \mathrm{TVPI}, \quad c^{\prime}:=\sum_{k=1}^{n} a_{k}^{\prime} x_{k} \leq d^{\prime} \in$ Poly, $a_{j}^{\prime} \neq 0$ and $a_{i} \cdot a_{i}^{\prime}<0$ then result $\left(c, c^{\prime}, x_{i}\right)=\left(\left|a_{i}^{\prime}\right| a_{j}+\left|a_{i}\right| a_{j}^{\prime}\right) x_{j}+$ $\left|a_{i}\right| \sum_{k \notin\{i, j\}} a_{k}^{\prime} x_{k} \leq\left|a_{i}^{\prime}\right| d+\left|a_{i}\right| d^{\prime}$ otherwise result $\left(c, c^{\prime}, x_{i}\right)=\perp$.

The $a_{j}^{\prime} \neq 0$ condition ensures $\left|\operatorname{vars}\left(\operatorname{result}\left(c, c^{\prime}, x_{i}\right)\right)\right|<\left|\operatorname{vars}\left(c^{\prime}\right)\right|$. Thus if $c^{\prime}$ is ternary then result $\left(c, c^{\prime}, x_{i}\right)$ is either binary or undefined. The partial result map can be lifted to a total map result $\left(I_{1}, I_{2}\right)$ for $I_{1} \subseteq$ TVPI and $I_{2} \subseteq$ Poly by analogy with definition 9 . With this in place, an operator extend $\left(I_{1}, I_{2}\right)$ is introduced, designed so that $\alpha\left(I_{1} \cup I_{2}\right) \models \operatorname{extend}\left(I_{1}, I_{2}\right)$ : 
Definition 14. The map extend $: \wp^{f}($ TVPI $) \times \wp^{f}($ Poly $) \rightarrow \wp^{f}($ TVPI $)$ is defined extend $(I, \delta)=\cup_{i \geq 0} I_{i}$ where $I_{0}=$ complete $(I \cup(\delta \cap$ TVPI $)), \delta_{0}=\delta \backslash$ TVPI, $R_{i+1}=\operatorname{result}\left(I_{i}, \delta_{i}\right), I_{i+1}=\operatorname{complete}\left(I_{i} \cup\left(R_{i+1} \cap \mathrm{TVPI}\right)\right)$ and $\delta_{i+1}=R_{i+1} \backslash I_{i+1}$.

If $\delta$ is comprised of ternary constraints, which is the dominating case [15], then result is applied once. If desired, incremental closure can be used to compute complete $\left(I_{i} \cup\left(R_{i+1} \cap \mathrm{TVPI}\right)\right)$, since proposition 2 extends to TVPI [8]. If extend $(I, \delta)$ is not logahedral, then $\alpha$ must subsequently be applied.

Example 5. Consider augmenting $I=\{x-y \leq 0,-x+y \leq 0\}$ with $c^{\prime}:=$ $x-2 y+z \leq 0$. Then $I_{0}=I$ and $\delta_{0}=\left\{c^{\prime}\right\}$. Thus $R_{1}=\operatorname{result}\left(I_{0}, \delta_{0}\right)=\{y+z \leq 0\}$ whence $\delta_{1}=\emptyset$ and $I_{1}=$ complete $\left(I_{0} \cup R_{1}\right)=I \cup\{y+z \leq 0, x+z \leq 0\}$. These inequalities cannot be inferred with the linear programming technique of [15].

Example 6. Let $I=\{w-x \leq 0, y-z \leq 0\}$ and $c^{\prime}:=w+x+y+z \leq 1$. Then $I_{0}=$ complete $(I)=I$ and $\delta_{0}=\left\{c^{\prime}\right\}$. Thus $R_{1}=\operatorname{result}\left(I_{0}, \delta_{0}\right)=\{2 w+y+z \leq 1$, $x+w+2 y \leq 1\}, I_{1}=I_{0}$ and $\delta_{1}=R_{1}$. Next $R_{2}=\operatorname{result}\left(I_{2}, \delta_{1}\right)=\{2 w+2 y \leq 1\}$, $I_{2}=$ complete $\left(I_{1} \cup R_{2}\right)=I_{1} \cup R_{2}=I \cup\{w+y \leq 1 / 2\}$ and $\delta_{2}=\emptyset$.

\subsection{Join}

Join is required to merge abstractions on different paths, as is shown with $P_{5}$ in section 3. If quotienting is omitted, then join over $\wp^{f}(\log )$ can be defined $I_{1} \sqcup I_{2}=\cup\left\{\bar{\exists} Y . I_{1} \sqcup_{Y} \bar{\exists} Y . I_{2}|Y \subseteq X \wedge| Y \mid=2\right\}$ where $J_{1} \sqcup_{Y} J_{2}=\alpha_{Y}\left(J_{1} \vee J_{2}\right)$ and $\checkmark$ is join (planar convex hull) for TVPI [15]. Join also benefits from completeness since if $I_{i}$ is complete then $\bar{\exists} Y I_{i}=\pi_{Y}\left(I_{i}\right)$. The TVPI operation $\vee$ is $O(n \lg n)$ where $n=\left|J_{1}\right|+\left|J_{2}\right|$, hence the overall cost of $\sqcup$ is dominated by completion.

With $\sqcap$ and $\sqcup$ thus defined, $\left\langle\wp^{f}(\log ), \mid=, \sqcap, \sqcup\right\rangle$ forms a lattice. Furthermore, $\log _{k}$ has additional structure since $C_{k}$ is finite (see definition 5). In particular, if $I \subseteq \log _{k}$ then there exists a finite $K \subseteq I$ such that $I \equiv K$. As a consequence $\left\langle\wp^{f}\left(\log _{k}\right), \models, \sqcap, \sqcup\right\rangle$ is a complete lattice.

Widening [6] is often applied with join in order to enforce termination. As has been explained elsewhere $[11,15]$, care must be taken not to reintroduce inequalities through completion that have been deliberately discarded in widening.

\section{Logahedra versus Octagons and TVPI}

Logahedra are theoretically well motivated and their representational advantages, as a generalisation of octagons, are of interest. To provide preliminary data on the power of bounded logahedra two sets of experiments were performed.

In the first experiment sets of integer points, $\{\langle x, y\rangle \mid x, y \in[-32,32]\}$ were randomly selected. For each set, between 1 and 63 points were generated. The best Oct $=\log _{0}$ and $\log _{k}$ (for $k \in[1,5]$ ) abstractions were computed and compared with the best TVPI abstraction. The comparison is based on the number of integer points in the abstractions. For example, one set of 23 points had 7 extreme points. The Oct, the five Log and the TVPI descriptions were satisfied 


\begin{tabular}{r|r|r|rrrrr} 
& & & \multicolumn{7}{|c}{$\log _{k}$} \\
vertices & sets & Oct & $\mathrm{k}=1$ & $\mathrm{k}=2$ & $\mathrm{k}=3$ & $\mathrm{k}=4$ & $\mathrm{k}=5$ \\
\hline 1 & 2044 & 0.000 & 0.000 & 0.000 & 0.000 & 0.000 & 0.000 \\
2 & 2958 & 82.640 & 43.941 & 30.675 & 26.396 & 25.024 & 24.481 \\
3 & 5923 & 1.874 & 0.998 & 0.700 & 0.611 & 0.584 & 0.576 \\
4 & 10423 & 0.557 & 0.294 & 0.195 & 0.163 & 0.153 & 0.149 \\
5 & 14217 & 0.352 & 0.192 & 0.125 & 0.100 & 0.092 & 0.089 \\
6 & 13550 & 0.276 & 0.152 & 0.097 & 0.075 & 0.067 & 0.064 \\
7 & 9058 & 0.234 & 0.131 & 0.081 & 0.061 & 0.054 & 0.051 \\
8 & 4345 & 0.205 & 0.115 & 0.071 & 0.053 & 0.046 & 0.043 \\
9 & 1508 & 0.188 & 0.105 & 0.064 & 0.047 & 0.040 & 0.038 \\
10 & 398 & 0.171 & 0.096 & 0.058 & 0.042 & 0.035 & 0.033 \\
11 & 64 & 0.165 & 0.095 & 0.054 & 0.037 & 0.031 & 0.029 \\
12 & 6 & 0.179 & 0.107 & 0.06 & 0.045 & 0.038 & 0.036
\end{tabular}

Table 1. Precision comparison of Oct and $\log _{k}$ against TVPI on random data

by $3221,3027,2881,2843,2835,2819,2701$ integer points. Thus the precision loss incurred by Oct over TVPI is $(3321-2701) / 2701=0.230$. Likewise the losses for Log relative to TVPI are $0.121,0.067,0.053,0.050$ and 0.044 . This was done for $64 \mathrm{~K}$ sets and the results are summarised in Table 1 . The table presents the mean precision loss where the sets are grouped by their number of vertices.

The data reveals that describing exactly two points is by far the most inaccurate scenario for Oct and $\log _{k}$; if the angle between the points is suitably acute then the TVPI constraints are satisfied by just the two points whereas the Oct and $\log _{k}$ constraints can be satisfied by a band of integral points. Precision loss decreases beyond this two point case. Enlarging the sample space does not noticeably effect the relative precision loss other than accentuating the two point case. Observe that the relative precision loss declines as $k$ increases, suggesting that logahedra can offer a significant precision improvement over octagons.

The second set of experiments compares the abstractions of two variable projections of the results of a polyhedral analysis tightened to integer points for a series of benchmarks $[1,2,13]$. As in the first experiment, comparisons are made between the number of points in the TVPI, Oct and $\log _{k}$ abstractions. Table 2 details benchmarks, their number of variables, the pair of variables in the projection, the number of integer points in the TVPI abstraction and the number of additional points for Oct and $\log _{k}$ abstractions. Benchmarks and projections where Oct and TVPI give the same abstraction are omitted from the table. This was the case for the additional 12 programs in the benchmark suite.

The data illustrates the power of octagons, with the majority of two variable projections being octagonal (indeed, many of these were intervals). It also illustrates that there are cases when non-octagonal constraints occur, with (as in the first experiment) $\log _{k}$ precision improving as $k$ increases.

Together, the results motivate further study. The random data demonstrates that logahedra have the potential to deliver precision gains over octagons, how- 


\begin{tabular}{rrr|r|rrrrr|r}
\multicolumn{1}{c}{ fixpoint vars project } & Oct & $\mathrm{k}=1$ & $\mathrm{k}=2$ & $\mathrm{k}=3$ & $\mathrm{k}=4$ & $\mathrm{k}=5$ & TVPI \\
\hline cars.inv & 5 & $(4,5)$ & 312 & 234 & 78 & 54 & 54 & 54 & 3640 \\
efm1.inv & 6 & $(3,6)$ & 1 & 0 & 0 & 0 & 0 & 0 & 128 \\
heap.inv & 5 & $(1,2)$ & 1056 & 0 & 0 & 0 & 0 & 0 & 33 \\
heap.inv & 5 & $(3,4)$ & 465 & 0 & 0 & 0 & 0 & 0 & 1055 \\
heap.inv & 5 & $(3,5)$ & 465 & 0 & 0 & 0 & 0 & 0 & 1055 \\
robot.inv & 3 & $(1,2)$ & 528 & 0 & 0 & 0 & 0 & 0 & 1089 \\
\hline scheduler-2p.invl1 & 7 & $(3,6)$ & 135 & 120 & 90 & 30 & 18 & 18 & 180 \\
scheduler-2p.invl1 & 7 & $(4,6)$ & 115 & 100 & 70 & 20 & 12 & 12 & 260 \\
scheduler-2p.invl1 & 7 & $(6,7)$ & 135 & 120 & 90 & 30 & 18 & 18 & 180 \\
scheduler-2p.invl2 & 7 & $(4,6)$ & 189 & 168 & 126 & 42 & 26 & 26 & 245 \\
scheduler-2p.invl2 & 7 & $(6,7)$ & 90 & 80 & 60 & 20 & 12 & 12 & 215 \\
scheduler-3p.invl1 & 10 & $(4,9)$ & 264 & 198 & 66 & 45 & 45 & 45 & 390 \\
scheduler-3p.invl1 & 10 & $(9,10)$ & 264 & 198 & 66 & 45 & 45 & 45 & 390 \\
scheduler-3p.invl3 & 10 & $(4,8)$ & 312 & 234 & 78 & 54 & 54 & 54 & 455 \\
scheduler-3p.invl3 & 10 & $(8,9)$ & 144 & 108 & 36 & 24 & 24 & 24 & 405 \\
scheduler-3p.invl3 & 10 & $(9,10)$ & 534 & 128 & 128 & 128 & 128 & 128 & 1725 \\
see-saw.inv & 2 & $(1,2)$ & 990 & 231 & 110 & 110 & 110 & 110 & 2454 \\
swim-pool-1.inv & 9 & $(4,6)$ & 62 & 61 & 59 & 55 & 47 & 31 & 4162
\end{tabular}

Table 2. Precision comparison of Oct and $\log _{k}$ against TVPI on analysis data

ever, the analysis data adds a note of caution. It is not surprising that many program invariants can be described by intervals, nor that many of the remainder are octagonal. The data (and the example in section 3 ) shows that the descriptive power of logahedra can improve analysis, leaving open the question of whether the additional cost (a higher constant in the complexity) pays for itself with improved accuracy. This answer to this question is in part application specific.

\section{Conclusion}

This paper has introduced logahedra, a new weakly relational abstract domain. A variant of logahedra, bounded $\operatorname{logahedra} \log _{k}$, where $k$ is the maximum exponent is also introduced. Logahedra lie strictly between octagons and TVPI in terms of expressive power, with octagons forming a special case, Oct $=\log _{0}$. Bounded logahedra retain the good computational properties of octagons, whilst being less restrictive. The theory for the abstract domain has been given, along with algorithms for each domain operation. Logahedra have the further advantage that their variable coefficients can be represented by their exponents, mitigating the problem of large coefficients that arise when using polyhedra or TVPI. A preliminary investigation into the expressive power of logahedra, plus a worked example, suggests that they can lead to more accurate analysis, but cautions that many invariants can be described using intervals and octagons. Future work will further investigate the application of logahedra in verification. 
Acknowledgements The authors thank Phil Charles, Tim Hopkins, Stefan Kahrs and Axel Simon for useful discussions. The work was supported by EPSRC projects EP/E033105/1 and EP/E034519/1. The authors would like to thank the University of St Andrews and the Royal Society for their generous support.

\section{References}

1. S. Bardin, A. Finkel, J. Leroux, and L. Petrucci. FAST: Fast Acceleration of Symbolic Transition Systems. In Computer Aided Verification, volume 2725 of LNCS, pages 118-121. Springer, 2003.

2. P. J. Charles, J. M. Howe, and A. King. Integer Polyhedra for Program Analysis. In $A A I M$, volume 5564 of $L N C S$, pages 85-99. Springer, 2009.

3. R. Clarisó and J. Cortadella. The Octahedron Abstract Domain. Science of Computer Programming, 64:115-139, 2007.

4. P. Cousot and N. Halbwachs. Automatic Discovery of Linear Restraints among Variables of a Program. In POPL, pages 84-97. ACM Press, 1978.

5. F. Eisenbrand and S. Laue. A Linear Algorithm for Integer Programming in the Plane. Mathematical Programming, 102(2):249-259, 2005.

6. N. Halbwachs. Détermination Automatique de Relations Linéaires Vérifiées par les Variables d'un Programme. PhD thesis, Université Scientifique et Médicale de Grenoble, 1979.

7. T. A. Henzinger, P.-H. Ho, and H. Wong-Toi. HyTech: A Model Checker for Hybrid Systems. Software Tools for Technology Transfer, 1:110-122, 1997.

8. J. M. Howe and A. King. Closure Algorithms for Domains with Two Variables per Inequality. Technical Report TR/2009/DOC/01, School of Informatics, City University London, 2009. http://www.soi.city.ac.uk/organisation/doc/ research/tech_reports/.

9. K. G. Larsen, F. Larsson, P. Pettersson, and W. Yi. Efficient Verification of Realtime Systems: Compact Data Structure and State-space Reduction. In IEEE RealTime Systems Symposium, pages 14-24. IEEE Computer Society, 1997.

10. F. Logozzo and M. Fähndrich. Pentagons: a Weakly Relational Abstract Domain for the Efficient Validation of Array Accesses. In ACM Symposium on Applied Computing, pages 184-188. ACM Press, 2008.

11. A. Miné. The Octagon Abstract Domain. Higher-Order and Symbolic Computation, 19(1):31-100, 2006.

12. C. G. Nelson. An $n^{\lg n}$ Algorithm for the Two-Variable-Per-Constraint Linear Programming Satisfiability Problem. Technical Report STAN-CS-78-689, Stanford University, Computer Science Department, 1978.

13. S. Sankaranarayanan, H. B. Sipma, and Z. Manna. Constraint Based Linear Relations Analysis. In $S A S$, volume 3148 of $L N C S$, pages 53-68. Springer, 2004.

14. S. Sankaranarayanan, H. B. Sipma, and Z. Manna. Scalable Analysis of Linear Systems Using Mathematical Programming. In Verification, Model Checking, and Abstract Interpretation, volume 3385 of LNCS, pages 25-41. Springer, 2005.

15. A. Simon. Value-Range Analysis of C Programs: Towards Proving the Absence of Buffer Overflow Vulnerabilities. Springer, 2008.

16. A. Simon and A. King. Exploiting Sparsity in Polyhedral Analysis. In C. Hankin, editor, SAS, volume 3672 of $L N C S$, pages 336-351. Springer, 2005.

17. A. Simon, A. King, and J. M. Howe. Two Variables per Linear Inequality as an Abstract Domain. In M. Leuschel, editor, Logic Based Program Development and Transformation, volume 2664 of LNCS, pages 71-89. Springer, 2002. 
Acknowledgements The authors thank Phil Charles, Tim Hopkins, Stefan Kahrs and Axel Simon for useful discussions. The work was supported by EPSRC projects EP/E033105/1 and EP/E034519/1. The authors would like to thank the University of St Andrews and the Royal Society for their generous support.

\section{References}

1. S. Bardin, A. Finkel, J. Leroux, and L. Petrucci. FAST: Fast Acceleration of Symbolic Transition Systems. In Computer Aided Verification, volume 2725 of LNCS, pages 118-121. Springer, 2003.

2. P. J. Charles, J. M. Howe, and A. King. Integer Polyhedra for Program Analysis. In $A A I M$, volume 5564 of $L N C S$, pages 85-99. Springer, 2009.

3. R. Clarisó and J. Cortadella. The Octahedron Abstract Domain. Science of Computer Programming, 64:115-139, 2007.

4. P. Cousot and N. Halbwachs. Automatic Discovery of Linear Restraints among Variables of a Program. In POPL, pages 84-97. ACM Press, 1978.

5. F. Eisenbrand and S. Laue. A Linear Algorithm for Integer Programming in the Plane. Mathematical Programming, 102(2):249-259, 2005.

6. N. Halbwachs. Détermination Automatique de Relations Linéaires Vérifiées par les Variables d'un Programme. PhD thesis, Université Scientifique et Médicale de Grenoble, 1979.

7. T. A. Henzinger, P.-H. Ho, and H. Wong-Toi. HyTech: A Model Checker for Hybrid Systems. Software Tools for Technology Transfer, 1:110-122, 1997.

8. J. M. Howe and A. King. Closure Algorithms for Domains with Two Variables per Inequality. Technical Report TR/2009/DOC/01, School of Informatics, City University London, 2009. http://www.soi.city.ac.uk/organisation/doc/ research/tech_reports/.

9. K. G. Larsen, F. Larsson, P. Pettersson, and W. Yi. Efficient Verification of Realtime Systems: Compact Data Structure and State-space Reduction. In IEEE RealTime Systems Symposium, pages 14-24. IEEE Computer Society, 1997.

10. F. Logozzo and M. Fähndrich. Pentagons: a Weakly Relational Abstract Domain for the Efficient Validation of Array Accesses. In ACM Symposium on Applied Computing, pages 184-188. ACM Press, 2008.

11. A. Miné. The Octagon Abstract Domain. Higher-Order and Symbolic Computation, 19(1):31-100, 2006.

12. C. G. Nelson. An $n^{\lg n}$ Algorithm for the Two-Variable-Per-Constraint Linear Programming Satisfiability Problem. Technical Report STAN-CS-78-689, Stanford University, Computer Science Department, 1978.

13. S. Sankaranarayanan, H. B. Sipma, and Z. Manna. Constraint Based Linear Relations Analysis. In $S A S$, volume 3148 of $L N C S$, pages 53-68. Springer, 2004.

14. S. Sankaranarayanan, H. B. Sipma, and Z. Manna. Scalable Analysis of Linear Systems Using Mathematical Programming. In Verification, Model Checking, and Abstract Interpretation, volume 3385 of LNCS, pages 25-41. Springer, 2005.

15. A. Simon. Value-Range Analysis of C Programs: Towards Proving the Absence of Buffer Overflow Vulnerabilities. Springer, 2008.

16. A. Simon and A. King. Exploiting Sparsity in Polyhedral Analysis. In C. Hankin, editor, SAS, volume 3672 of $L N C S$, pages 336-351. Springer, 2005.

17. A. Simon, A. King, and J. M. Howe. Two Variables per Linear Inequality as an Abstract Domain. In M. Leuschel, editor, Logic Based Program Development and Transformation, volume 2664 of LNCS, pages 71-89. Springer, 2002. 